\title{
PROKLA-Redaktion
}

\section{Editorial: Kritik der Wirtschaftswissenschaften}

Die Finanzkrise der Jahre 2008/2009 und die folgende Wirtschaftskrise hat nicht nur die Ökonomie, sondern auch die herrschende ökonomische Theorie erschüttert. Weitgehend deregulierte Finanzmärkte, nach neoklassischer Lehre der Gipfel ökonomischer Effizienz, stürzten in kürzester Zeit in eine tiefe Krise. Nur durch umfangreiche staatliche Bürgschaften und Finanzspritzen für Großbanken - ganz im Widerspruch zu den bis dato weithin geteilten marktradikalen Glaubenssätzen - konnte ein weitgehender Zusammenbruch des internationalen Finanzsystems verhindert werden. Die analytische Hilflosigkeit der Neoklassik war angesichts der Krise offensichtlich, so manche Auftritte ihrer Vertreter wirkten nur noch peinlich. Angesichts der enormen Konjunkturprogramme, mit denen die USA, Deutschland und andere Staaten auf die Finanzmarkt- und Wirtschaftskrise reagierten, sahen manche Beobachter schon eine Renaissance des Keynesianismus am Horizont. In manchen Medien erinnerte man sich sogar wieder der Marxschen Theorie, die immer schon argumentiert hatte, dass Krisen notwendigerweise zum Kapitalismus gehören.

Geschadet hat dies alles der Neoklassik aber nicht sehr viel. Der Ruf ist zwar etwas angeschlagen. Dass der Markt die Beste aller möglichen Welten liefert, dass die Privatisierung staatlicher Unternehmen automatisch zu mehr Effizienz und höherem Wohlstand führe, lässt sich in der Öffentlichkeit nicht mehr genauso leicht vertreten wie noch vor ein paar Jahren. Heterodoxe, keynesianische und an Marx orientierte Stimmen sind etwas lauter geworden. Aber institutionell, an Hochschulen, in Forschungsinstitutionen, in Beraterstäben ist die Dominanz der Neoklassik ungebrochen - sowohl auf der „Angebots“- als auch auf der "Nachfrageseite“.

Seit Jahren steht die Betriebswirtschaftslehre in der Beliebtheit der Studierenden an deutschen Hochschulen unangefochten auf Platz eins. Mehr junge Menschen denn je ziehen sie den Rechtswissenschaften oder der Medizin vor und selbst bei jungen Frauen rangiert sie inzwischen weit vor der früher favorisierten Germanistik. Die wenigsten der angehenden Manager, Finanz- und Unternehmensberater entscheiden sich aus Neigung oder Interesse für dieses Studium, sondern meist, weil sie sich davon prestigeträchtige Berufe und ein relativ hohes Einkommen versprechen. Innerhalb der Wissenschaftscommunity wird dem Fach der wissenschaftliche Charakter zwar oftmals abgesprochen, da weite Teile davon weder theoretisch noch empirisch höhere Ansprüche verfolgen, sondern in erster Linie auf Klassifizierungen und Handlungsempfehlungen ausgerichtet sind. Häufig werden etwa „Kundenorientierung“, „Intrapreneurship“, „Corporate Branding" oder "Guerilla-Marketing" als Patentlösungen vermarktet, ohne auf die Grenzen und inneren Widersprüche derartiger Konzepte auch nur ansatzweise einzugehen. Die MBA-Ausbildung (Master of Business Administration), quasi die 
Königsdisziplin der Manager-Ausbildung, wird selbst intern von manchen kritisiert, weil komplexe Realitäten auf Kennzahlen reduziert werden, die schnelle Entscheidungen ohne Kenntnis des wirtschaftlichen und sozialen Kontextes ermöglichen sollen. Genauso unkritisch wie solche Inhalte von vielen Lehrenden präsentiert werden, werden sie auch von den meisten Studierenden akzeptiert.

Die Popularität des Fachs ist Symptom eines gesellschaftlichen Zustandes, in dem betriebswirtschaftliche Rezepturen nicht auf die Gestaltung der Wirtschaft beschränkt bleiben, sondern ebenso auf Kultur und Bildung oder den Sozialstaat angewendet werden sollen - die Tendenz zur „Verbetriebswirtschaftlichung" wurde bereits in PROKLA 148 (2007) thematisiert. Die Akteure in den Mittelpunkt zu rücken und Märkte als effizienteste Form für die Allokation von Ressourcen zu propagieren, gehört für die Volkswirtschaftslehre zum Traditionsbestand des Faches. Doch war die fortgesetzte Dominanz der Neoklassik in den letzten Jahrzehnten auch mit einer Neugewichtung wirtschaftswissenschaftlicher Schwerpunkte verbunden. Gegenüber der Makroökonomie, die in ihren keynesianischen Varianten stets im Verdacht stand, den Weg des reinen Glaubens an den Markt ein Stück weit zu verlassen, hat die Mikroökonomie stark an Terrain gewonnen. Dabei hat sich die Mikroökonomie durchaus als lernfähig erwiesen hat, indem sie sich vom Idealtyp des homo oeconomicus, dieser unablässig ständig ihren Nutzen oder Gewinn kalkulierenden Rechenmaschine auf zwei Beinen, weitgehend verabschiedet hat. Dass Menschen über begrenzte Informationen verfügen, in ihrem Verhalten von anderen abhängen und kulturelle Prägungen aufweisen, war in der Soziologie seit jeher Gemeingut, hat sich nun aber auch bei den behavioral econo- mics herumgesprochen und Spezialisierungen wie die behavioral finance bieten neue Erkenntnisse zum Verhalten der Akteure auf den Finanzmärkten an. Die Einsicht, dass sich diese Akteure keineswegs immer zweckrational verhalten (können), sondern unter Umständen „risikoavers“ sind, für Informationen ein "cognitive framing“ anwenden und häufig ein „Herdenverhalten" an den Tag legen, bedeutet zweifellos eine Annäherung an die Realität. Dennoch erweisen sich derartige Erklärungen, mit denen Zusammenhänge der Gesellschaft auf „irrationale" psychische Dispositionen ihrer Mitglieder reduziert werden, als halbherzig, da sie die strukturellen Momente kapitalistischen Wirtschaftens ausblenden - etwa den Druck, unter dem viele dieser Akteure stehen, auf deregulierten Märkten und unter der Peitsche der Konkurrenz kurzfristig Profite zu maximieren. Zweifellos handeln sie dabei unter Unsicherheit, können aber - soweit sie wie Banken über eine gewisse Machtposition verfügen - davon ausgehen, dass es ihnen gelingen wird, die Risiken fehlgeschlagener Kalküle auf schwächere Marktteilnehmer oder den Staat abzuwälzen. Insofern wären nicht nur systemische $Z$ wänge der Orientierung am Profit, sondern auch unterschiedliche Handlungsspielräume der Akteure und gesellschaftliche Machtverhältnisse zu analysieren, Dimensionen, die von der modernisierten Mikroökonomie geflissentlich ausgeblendet bleiben, wenn sie von einer Bilderbuchwelt formal und faktisch gleichgestellter Akteure ausgeht.

Dabei ist der Vorwurf der Realitätsferne an die Adresse der dominanten Volkwirtschaftslehre alles andere als neu. Hans Albert erhob ihn bereits in den 1960er Jahren gegenüber einer Wissenschaft, die sich in der Konstruktion von eleganten Modellen gefiel, in denen sämtliche Akteure stets rational handelten und die 
Wirtschaft ein System von Anpassungen und wiederkehrenden Zuständen des Gleichgewichts darstellte, das allenfalls von außen gestört werden konnte - er bezeichnete diese Realitätsverweigerung als „Modellplatonismus“. Gekontert wurden solche Argumente mit der Behauptung, man befinde sich halt erst am Anfang eines noch langen, aber durchaus erfolgversprechenden Forschungsweges - eine Behauptung, die auch noch vier Jahrzehnte und mehrere Krisen später mit dem gleichen Recht wiederholt wird.

Wesentlich stärker als die bloß formale Kritik am Modellplatonismus ist eine Kritik, die sich inhaltlich auf die Neoklassik bezieht. Dazu gibt es prinzipiell zwei unterschiedliche Ansatzpunkte. Man kann einerseits eine an der Marxschen Kritik der politischen Ökonomie orientierte Kritik neoklassischer Grundkategorien leisten oder aber immanent aufzeigen, dass die Neoklassik nicht einmal ihre eigenen Erkenntnisansprüche erfüllen kann: Für die Gültigkeit zentraler Aussagen der Neoklassik bedarf es nicht nur der üblichen, wirklichkeitsfremden Abstraktionen, die oben schon erwähnt wurden, man muss noch weitergehen und eine „Ein-GutÖkonomie“voraussetzen (d.h. eine Ökonomie, in der mit nur einer einzigen Art von Kapitalgütern produziert wird). Bereits bei einer "Zwei-Gut-Ökonomie“ erweisen sich beliebte Lehrsätze als falsch, wie etwa der, dass Reallohnsatz und Umfang der Beschäftigung in einem inversen Verhältnis zueinander stehen, woraus dann abgeleitet wird, dass der Beschäftigungsstand nur erhöht werden kann, wenn die Lohnsätze (oder auch die Lohnnebenkosten) sinken.

Deutlich wurden solche Defizite in der sogenannten Cambridge-CambridgeDebatte (Cambrigde USA versus Cambridge England), die in den 1960er Jahren auf die Veröffentlichung von Piero Sraffas
Production of Commodities by Means of Commodities folgte. Weithin bekannt sind die logischen Inkonsistenzen der neoklassischen Theorie deshalb aber noch lange nicht. Hans-Peter Büttner resümiert in seinem Beitrag die zentralen Punkte dieser Kritik an der Preis-, Kapital- und Wettbewerbstheorie. Allerdings blieb auch sie für die Neoklassik weitgehend folgenlos. Derselbe Paul Samuelson, der als Hauptvertreter der Neoklassik in der Cambridge-Cambridge-Debatte in einem Zeitschriftenbeitrag einräumen musste, dass bis dahin vertretene zentrale Positionen nicht mehr haltbar sind, legte die widerlegten Positionen sowohl vor als auch nach dieser Kontroverse seinem auch heute noch einflussreichen Lehrbuch der Volkswirtschaftslehre zugrunde. Dass die Studierenden der Volks- oder Betriebswirtschaftslehre während ihres Studiums überhaupt etwas von der Existenz der Debatte und den Inkonsistenzen der Neoklassik hören, ist eine eher seltene Ausnahme. Im akademischen Unterricht und in den allermeisten Lehrbüchern ist es verpönt sich mit solchen Fragen auseinanderzusetzen.

Das zeigt sich deutlich, wenn Hanno Pahl die Gattung der nationalökonomischen Lehrbücher unter die Lupe nimmt. Bemerkenswert an ihnen ist sowohl ihr hoher Grad an Standardisierung wie auch, dass sie meist zwar nicht dem Forschungsstand der Disziplin entsprechen, dennoch entscheidend dazu beitragen, den hegemonialen Wissensstand als Kanon abzusichern und zu legitimieren. Diese Textbook Economics markieren nicht nur den Wissenshorizont der Studierenden der Volkswirtschaftslehre, so wie er in den einschlägigen Modulen vorgezeichnet wird, sondern ebenso denjenigen der eingangs genannten immer zahlreicheren Studierenden der Betriebswirtschaftslehre, die angesichts der Überfülle an Stoff in den 
übrigen Fächern noch weniger Chancen haben, hierzu jemals eine kritische Distanz zu finden.

Weitere Bausteine zur Erklärung für die institutionelle Absicherung der Neoklassik werden von Leonhard Dobusch und Jakob Kapeller bereitgestellt. Sie untersuchen das Verhältnis von Orthodoxen und Hetererodoxen in der ökonomischen Forschung und Lehre. Die Gegenüberstellung dieser beiden Gruppen knüpft an historische Kämpfe der katholischen Kirche gegen Abweichler und Ketzer an. Wenn auch der Scheiterhaufen heutzutage zur Ausgrenzung Andersdenkender nicht mehr üblich ist, so erweisen sich die Methoden des orthodoxen Mainstreams gleichwohl ebenfalls als äußerst wirksam: die Verhinderung von Pluralismus und offenen Debatten durch schlichtes Ignorieren der Heterodoxen, insbesondere durch Nicht-Zitieren in wissenschaftlichen Journalen und durch Nicht-Berufungen auf einschlägige Lehrstühle. Auch die beliebten Zeitschriften-Rankings zeigen hier einen weiteren Verstärkungs-Effekt des „methodischen Monismus“ im Rahmen des vorherrschenden Paradigmas. $\mathrm{Zu}$ den Abweichungen, die der Mainstream allenfalls mit scheelem Blick betrachtet, gehört auch die von Katharina Mader und Jana Schultheiss portraitierte feministische Ökonomie, so wie sie sich seit den 1970er Jahren entwickelt hat. Die Autorinnen betonen, dass diese kein geschlossenes System darstellt, sondern eine große Variationsbreite aufweist, da ihr verschiedene feministische wie ökonomische Ansätze zugrunde liegen. Dennoch gibt es einen gemeinsamen Ausgangspunkt, nämlich die Kritik an der Vorstellung, Wirtschaft sei geschlechtsneutral. Sobald diese unzulässige Vereinfachung aufgegeben wird, erscheinen bisherige Leerstellen in einem neuen Licht - etwa formelle und informelle
Macht- und Herrschaftsverhältnisse oder die Frage der Reproduktion einer Gesellschaft und der damit verbundenen Arbeit - Ergebnisse, die sich bisher vor allem im Bereich der Arbeitsmarktökonomie als schlüssig erwiesen haben und bei denen es sich keineswegs nur um "Frauenthemen“ handelt, sondern mit denen allgemeine Unzulänglichkeiten der herrschenden ökonomischen Theorie aufgedeckt werden.

Die Dominanz der Neoklassik hat nicht nur Bedeutung für das akademische Leben, sondern auch für die reale wirtschaftliche Praxis von Staat und Unternehmen, wie in drei ausgewählten Bereichen gezeigt wird. Katrin Hirte und Walter Ötsch fragen danach, wie sich der jeweilige ökonomische Mainstream in den Gutachten des „Sachverständigenrats zur Begutachtung der gesamtwirtschaftlichen Entwicklung" Deutschlands (SVR), dem wichtigsten wirtschaftspolitischen Beratungsgremium der Bundesregierung, niederschlägt. Unter anderem haben bestimmte Lehrer-SchülerVerhältnisse an den Universitäten dafür gesorgt, den monolithischen Charakter der Volkswirtschaftslehre zu befestigen und als Grundlage für wirtschaftspolitische Weichenstellungen der Bundespolitik zu legitimieren. Vanessa Redak widmet sich der besonders engen Verschränkung zwischen universitärer Forschung, dem Wandel der Bankenpraxis und der Politik von Aufsichtsbehörden, wie sie seit den 1990er Jahren für den Bereich der Finanzmärkte entstanden ist. Der Siegeszug der "quantitativen Risiko-Messmodelle" wurde von der Wissenschaft befördert, durch Nobelpreise mit höheren Weihen versehen und durch Aufsichtsbehörden institutionell verankert. Dieser Prozess hat innerwissenschaftlich zwar auch eine Reihe von kritischen Stimmen auf den Plan gerufen, die methodische Mängel und erhebliche systemische Probleme der zunehmenden Verbreitung 
solcher Modelle nachgewiesen haben; das dramatische Versagen dieser Modelle wurde spätestens in der weltweiten Finanzkrise der letzten Jahre mehr als deutlich. Trotz aller gegenläufiger Erfahrung gelten "Stresstests“ der Politik jedoch nach wie vor als bevorzugtes Mittel zur Bändigung der Finanzmärkte. Luise Görges und Ulf Kadritzke greifen einen weiteren shooting star der neueren wirtschaftswissenschaftlichen Diskussion und unternehmerischen Praxis auf, die „Unternehmensethik“ oder corporate social responsibility. International agierenden Konzernen wurde in den letzten Jahrzehnten durch non-governmental organizations zunehmend öffentlichkeitswirksam vorgehalten, sie würden ökologische und soziale Standards sowie Menschenrechte missachten. Die Konzerne reagierten auf ihre Weise, indem sie Selbstverpflichtungen eingingen, gelegentlich punktuelle Verbesserungen vornahmen, vor allem aber mit wissenschaftlicher Begleitung eine
CSR-Industrie ins Leben riefen, die ihnen laufend Persilscheine ausstellt. Dies wirft auch für dieses modische Aktivitätsfeld die Frage auf, inwieweit Gesellschaften es einigen wenigen, wirtschaftlich äußerst mächtigen Akteuren überlassen wollen, die Interessen und Angelegenheiten ihrer Stakeholder nach eigenem Gutdünken in unternehmerische Strategien aufgehen zu lassen und gleichzeitig den Abbau staatlicher und überstaatlicher Regelungen weiter zu forcieren.

Außerhalb des Schwerpunkts schließt Philippe Kellermann mit seinem Beitrag an die in PROKLA 160 und 162 zwischen Jan Schlemermeyer und Alex Demirović geführte Debatte über Agnolis „Kritik der Politik“ an. Gegenüber dieser Debatte nimmt Kellermann allerdings einen interessanten Perspektivenwechsel vor, indem er die libertären Positionen, die Agnoli Marx zuschreibt, viel eher im Anarchismus eines Bakunin verortet.

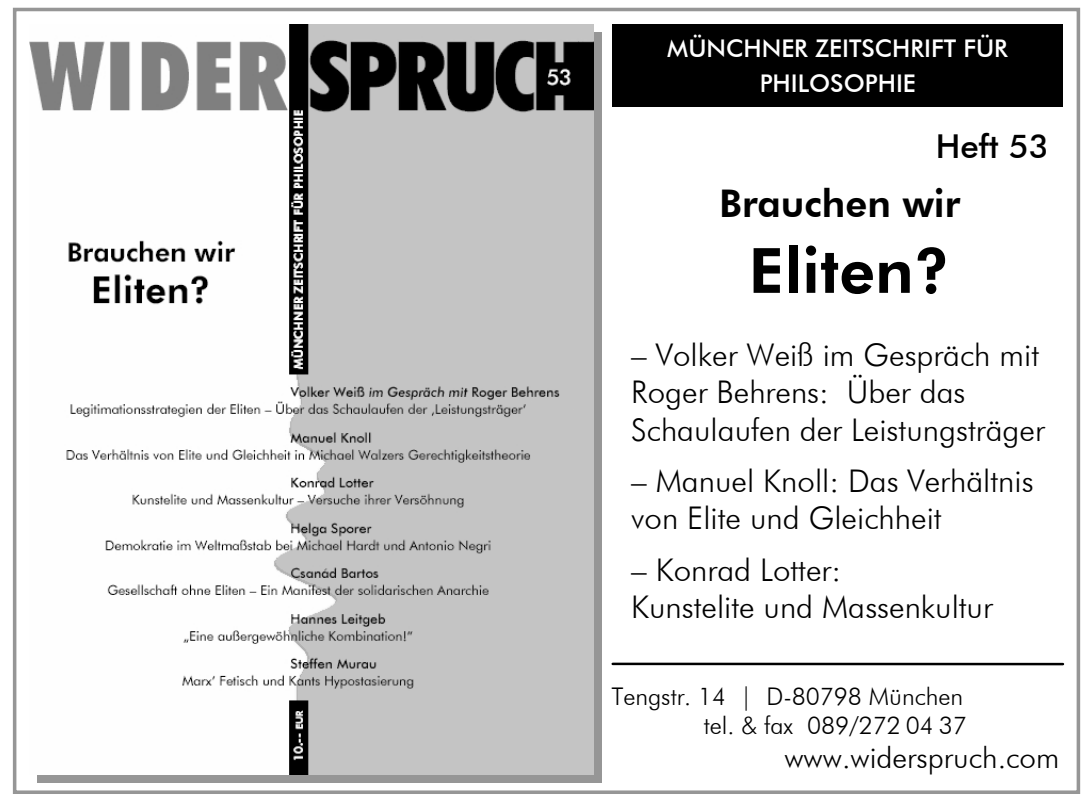




\section{PROKLA 165 Gesellschaftstheorie und Gesellschaftskritik heute (Dezember 2011)}

Im Anschluss an Marx wurden zwar die kapitalistischen Produktionsverhältnisse vielfach untersucht, der Zusammenhang zwischen der Ebene der Produktionsverhältnisse und den Ungleichzeitigkeiten der Entwicklung der jeweiligen Gesellschaften, den Klassenstrukturen, Geschlechterverhältnissen, Formen politischer Herrschaft, den Selbstverhältnissen der Individuen etc. wurde häufig nur mehr oder weniger oberflächlich aufgegriffen. Erst in den letzten Jahrzehnten wurde dieser Zusammenhang verstärkt zum Thema gemacht, doch scheint es noch immer schwierig zu sein die Kluft zu überwinden. Während sich die Kritik der politischen Ökonomie in der Analyse der jüngsten Finanzmarkt- und Wirtschaftskrisen durchaus auf der Höhe der Zeit befindet, bleibt die Analyse der gegenwärtigen sozialen Verhältnisse, Entwicklungsalternativen und Konflikte weit zurück. Die Debatten über die Kritik der politischen Ökonomie, über verschiedene Formen der Ungleichheit, über Geschlechterverhältnisse, Rassismus, über sexuelle Orientierungen, über Jugendkulturen, Alltagswelten oder Formen der Sozialkritik verlaufen weitgehend getrennt voneinander.

Materialistische Gesellschaftstheorie und -kritik muss aber versuchen, den Zusammenhang der verschiedenen Formen von Herrschaft ebenso wie die Möglichkeiten der Emanzipation zu analysieren. Welche Begrifflichkeiten, welches Verhältnis von Theorie und Kritik ist aber dazu nötig? Wie ist heute am Subjekt als Bezugspunkt emanzipatorischer Praxis anzusetzen? Was ist heute materialistische Gesellschaftstheorie und in welchem Verhältnis steht sie zu dem weiten Feld poststrukturalistischer Theorien? Was macht gegenwärtig Ansätze wie die von Rancière, Żiżek, Badiou so attraktiv? Die Diskussion über diese und verwandte Fragen soll in dem Heft geführt werden.

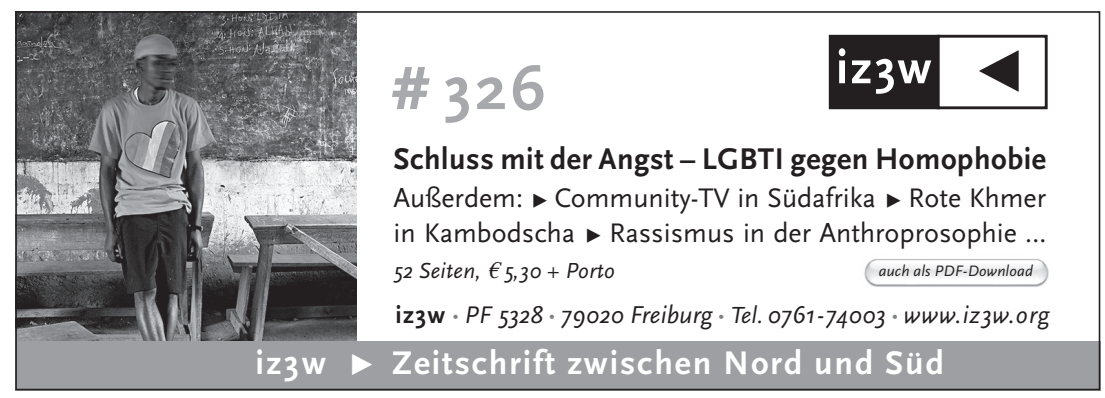




\section{Jürgen Habermas: Wie demokratisch ist die EU?}
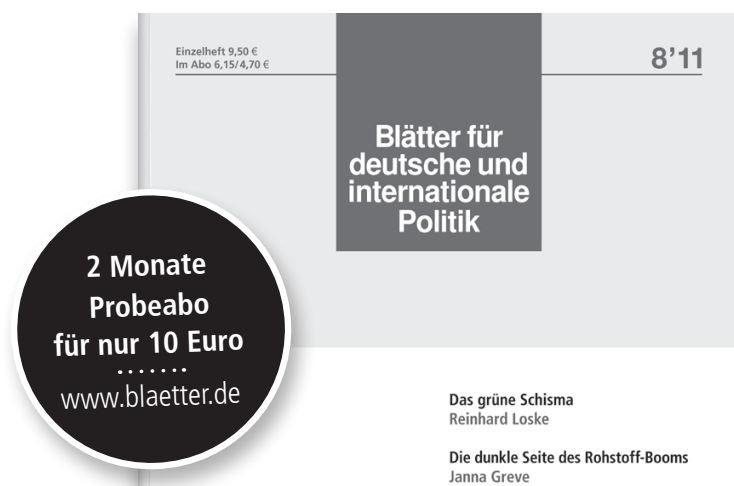
Russlands zweite Indien: Arbeit für alle?
Karl Schlögel
Wie demokratisch
ist die EU?
Jürgen Habermas

\section{Das Recht auf die Stadt Andrej Holm \\ Das grüne Schisma Reinhard Loske \\ Die dunkle Seite des Rohstoff-Booms Janna Greve \\ Von der Pluralität zur Hegemonie \\ Literarische Grenzziehungen: \\ Die Schriftsteller und der Mauerbau}

\section{(1)}

\section{Testen Sie die »Blätter»} www.blaetter.de|030/3088-3644|abo@blaetter.de

Herausgegeben von: Seyla Benhabib, Peter Bofinger, Micha Brumlik, Jürgen Habermas, Detlef Hensche, Rudolf Hickel, Claus Leggewie, Jens Reich, Saskia Sassen, Friedrich Schorlemmer u.a. 